\title{
Clostridium perfringens in London, July 2009: two weddings and an outbreak
}

J Eriksen ${ }^{1,2,3}$, D Zenner (Dominik.Zenner@hpa.org.uk)1,3,4,5, S R Anderson ${ }^{4}$, K Grant $^{6}$, D Kumar

1. Health Protection Agency, Centre for Infections, London, United Kingdom

2. European Programme for Intervention Epidemiology Training (EPIET), European Centre for Disease Prevention and Control (ECDC), Stockholm, Sweden

3. These authors contributed equally to the work and share first authorship

4. North West London Health Protection Unit, London, United Kingdom

5. London School of Hygiene and Tropical Medicine, London, United Kingdom

6. Gastrointestinal, Emerging and Zoonotic Infections Department (GEZI) - Laboratory of Gastrointestinal Pathogens (LGP), HPA Centre for Infections, London, United Kingdom

Eriksen J, Zenner D, Anderson SR, Grant K, Kumar D. Clostridium perfringens in London, July 2009: two weddings and an outbreak. Euro Surveill.

2010;15(25):pii=19598. Available online: http://www.eurosurveillance.org/ViewArticle.aspx?Articleld=19598

Article published on 24 June 2010

Food poisoning outbreaks caused by Clostridium perfringens enterotoxin occur occasionally in Europe but have become less common in recent years. This paper presents the microbiological and epidemiological results of a large $C$. perfringens outbreak occurring simultaneously at two weddings that used the same caterer. The outbreak involved several London locations and required coordination across multiple agencies. A case-control study $(n=134)$ was carried out to analyse possible associations between the food consumed and becoming ill. Food, environmental and stool samples were tested for common causative agents, including enterotoxigenic $C$. perfringens. The clinical presentation and the epidemiological findings were compatible with $C$. perfringens food poisoning and $C$. perfringens enterotoxin was detected in stool samples from two cases. The case-control study found statistically significant associations between becoming ill and eating either a specific chicken or lamb dish prepared by the same food handler of the implicated catering company. A rapid outbreak investigation with preliminary real-time results and the successful collaboration between the agencies and the caterer led to timely identification and rectification of the failures in the food handling practices.

\section{Background}

Food poisoning caused by $C$. perfringens is quite common [1]. Occasional outbreaks occur in Britain [2]; however, due to symptoms often being mild and of short duration, outbreaks are often not reported. We report the unusual occurrence of a simultaneous outbreak of C. perfringens at two large venues in London in July 2009.

Gastrointestinal illness caused by $C$. perfringens is characterised by sudden onset of abdominal pain followed by diarrhoea, and less commonly by vomiting and fever. Severe illness and fatal outcomes are rare. A short incubation period is usual (median 10-12 hours, range 6-24 hours [3]). Disease symptoms are caused by an enterotoxin produced by $C$. perfringens type $A$ strains. Sufficient heat inactivates $C$. perfringens vegetative cells, however, its spores can survive and germinate in contaminated food under circumstances of poor temperature control, particularly a lack of cooling and insufficient reheating [4]. If food containing high numbers $\left(>10^{5} \mathrm{cfu} / \mathrm{g}\right)$ of $C$. perfringens vegetative cells is consumed, the bacterial cells can sporulate and produce enterotoxin in the human small intestine. Most C. perfringens food poisoning outbreaks are caused by a failure of adequate food preparation procedures. Recent evidence has also shown that healthy human food handlers can carry enterotoxigenic $C$. perfringens indicating that poor personal hygiene in catering staff is a risk factor for this foodborne illness [5].

In July 2009, the North West London Health Protection Unit (NWL HPU) was notified of a number of cases of gastroenteritis, which appeared to have been contracted at two different weddings on the same day in different London boroughs (administrative districts). The food at both these events had been supplied by the same caterer from a third borough.

The outbreak investigation and control was led by the local Health Protection Unit (HPU) in collaboration with the environmental health departments of three London boroughs. This team was supported by several divisions of the Health Protection Agency (HPA), including the regional Food, Water and Environmental laboratory, the National Reference Laboratory at the Centre for Infections, and the Regional Press Officers.

This paper adds to the current evidence base on C. perfringens outbreaks and reports on the findings of the microbiological and epidemiological investigations, as well as the logistics of investigating such outbreaks. 


\section{Methods}

\section{Case definitions}

Probable cases were defined as persons who fell ill with one or more of the following symptoms: abdominal pain, diarrhoea or vomiting within 24 hours of attending a wedding in either of the affected venues. Confirmed cases were defined as persons fulfilling the case definition with microbiological confirmation of a gastroenteritis-causing organism in their stool sample. Controls were defined as persons who attended either of the two wedding receptions but did not develop any of the above symptoms within the following 24 hours.

\section{The case-control study}

Case-control methodology was used to investigate the outbreaks. A cohort study could not be conducted because complete guest lists were not available from either of the two events. Verbal consent was obtained from adults and young people. Children under the age of 12 years were excluded from the study, as their food histories were unlikely to be accurate. Many symptomatic people notified themselves, but active case finding was performed through the wedding hosts and environmental health officers. Controls were nominated by cases. Ninety-three cases and 41 controls were identified.

A single standardised questionnaire including questions on food consumption was administered through telephone interviews $(n=124)$ or in person $(n=10)$ between day 2 and day 9 after the wedding. The questionnaire had been developed, piloted and tested for validity in other outbreaks prior to this incident. Some common food items were served at the two weddings and the same questionnaire was used with the food items adapted for the specific venues. The data was entered into a secure database, cleaned and crosschecked for inconsistencies. Data analysis was performed with Intercooled STATA 9.2. Odds ratios (OR) and confidence intervals $(\mathrm{CI})$ were calculated for all

\section{FIGURE 1}

Incubation period, gastroenteritis outbreak at two weddings (venues A and B), London, July $2009\left(n=91^{\mathrm{a}}\right)$

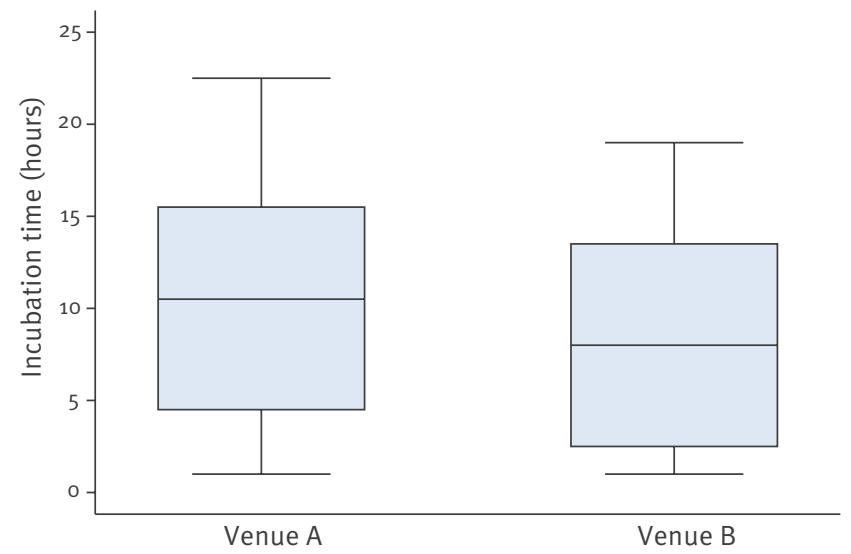

a For two cases onset time was not known. food items and chi-square and Fisher's exact tests were used for single variable analysis. Multivariable analysis was performed using logistic regression. The model was built in a forward fashion, and items which were significantly associated with illness in the single variable analysis ( $p<0.05)$ were included stepwise according to their a priori plausibility. At each step, a likelihood ratio (LR) test was performed to test whether the new variable significantly added to the explanatory power of the model. Only variables achieving an LR test of $p<0.05$ were kept in the model. Continuous variables were compared with two-sided t-tests, using natural logarithms to transform skewed distributions if appropriate.

Single- and multivariable analysis of all served food and drink items was performed separately for both venues. In addition the data from the two venues was merged for an analysis of all food items, treating the two wedding receptions as one large outbreak. In this analysis common food items, served at both venues and according to the caterer prepared together, were analysed as a single common variable. The two separate analyses had similar results in the final model and only results of the common analysis are shown in this paper, as these are based on a larger sample.

The environmental health officers collected detailed information on the preparation, storage, and transportation processes for the food catered at the two events.

\section{Microbiology}

Stool samples from eight symptomatic patients were collected and tested for a range of routine organisms, including Campylobacter, Salmonella and Shigella, and norovirus. Five of these were also tested for the presence of $C$. perfringens enterotoxin by Techlab ELISA at the Health Protection Agency (HPA) Laboratory of

\section{FIGURE 2}

Gastroenteritis outbreak at two weddings (venues A and B), London, July $2009\left(\mathrm{n}=91^{\mathrm{a}}\right)$

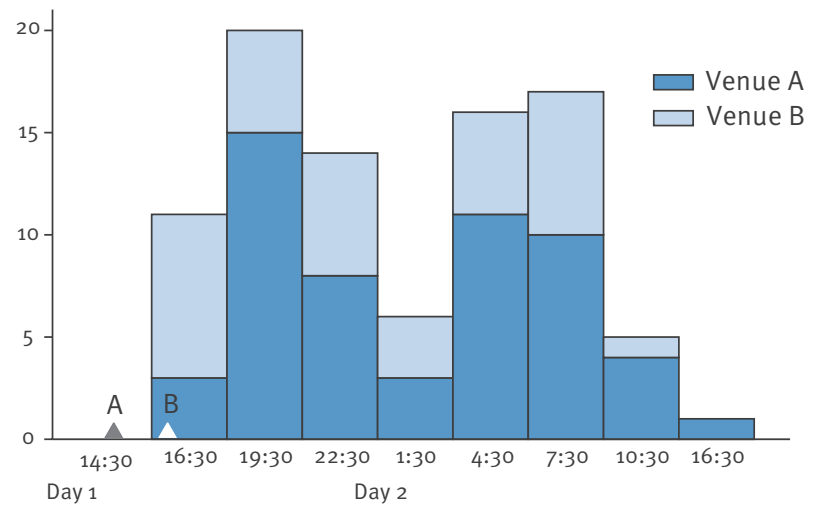

Scale: three-hourly.

The triangles indicate the start of the meals in venue $A(A)$ and venue $B(B)$, respectively.

a For two cases onset time was not known. 
Gastrointestinal Pathogens, Centre for Infections. Samples from leftover food and environmental swabs from the catering company, in addition to stool samples and hand swabs from the catering staff were sent and tested at the HPA's Food Water and Environmental Laboratory, Centre for Infections.

\section{Results}

A total of 134 individuals were interviewed from the two wedding receptions (referred to as venue $A$ and venue $B$ ), of whom 93 were cases ( 57 and 36 from venue $A$ and venue $B$, respectively) and 41 were controls (16 and 25 from venue $A$ and venue $B$, respectively). An estimated 150 guests attended venue $A$ and about 400 attended venue $B$.

\section{Descriptive epidemiology}

The median age of cases was 28 years (mean: 31.5 years; range: 12-74 years). Males and females were almost equally distributed ( $55 \%$ and $45 \%$ respectively). The majority of cases had a rapid onset of symptoms and the median incubation period was 9.5 hours, (mean: 10 hours; range: 1-22.5 hours), however, the incubation period in venue $B$ was significantly shorter than in venue $A$ ( 8 hours versus 10.5 hours, respectively, $p=0.033$, Figure 1 ). The epidemiological curve is shown in Figure 2. The median duration of illness was two days (mean: 2.3 days; range: 1-10 days).

Overall, the majority of the 93 interviewed cases experienced symptoms of diarrhoea (95\%) and abdominal pain $(89 \%)$, followed by nausea $(51 \%)$, headaches (38\%), vomiting (24\%) and fever (18\%). Three patients reported to have had blood in their stools, an uncommon event in $C$. perfringens gastroenteritis. All three had attended venue $A$. Significantly more cases in venue $B$ experienced vomiting compared to venue $A$ ( $39 \%$ versus $14 \%$, Fisher's exact test: $p=0.001$ ). There were no other significant differences in the epidemiological characteristics or the symptoms among the cases at the two venues.

The single variable analysis of all food and drinks items in both venues is shown in Table 1. However, many people will have chosen more than one dish in this buffet-style menu. In order to adjust for confounding, a logistic regression analysis was performed. Table 2 shows the final logistic regression model and all candidate variables which were excluded because they did not add to the explanatory power of the model.

After adjusting for all other food or drinks items, only the Jeera chicken (OR: 11.5; 95\% Cl: 3.7-35.9) and the Lamb karahi (OR: $2.71 ; 95 \% \mathrm{Cl}: 1.11-6.58$ ) remained in the final model and were significantly associated with illness. These two dishes accounted for $88.2 \%$ of illnesses. In all, $92 \%(n=48)$ of persons who ate the Jeera chicken and $75 \%(n=69)$ of those who ate the Lamb karahi became ill. Thirty-five of the 37 who consumed both of these dishes became ill. There was no statisti- cal interaction between the lamb and the chicken dish $(p=0.995)$.

\section{Microbiology \\ Stool samples from cases}

All eight stool samples available for testing were negative for all routinely tested organisms, i.e. Salmonella, Shigella, Campylobacter and enteric viruses. Five of these samples were tested for $C$. perfringens enterotoxin and it was detected in two.

Leftover food samples

A small amount of uneaten food was collected from take-home portions of attendees from both weddings. From venue $B$, only samples of the Daal tarka (a lentil dish) and an unspecified lamb dish (several lamb dishes were served at venue $B$ ) remained, and in both of them Enterobacteriaceae and Bacillus cereus were detected at levels exceeding the acceptable thresholds $\left(>10^{5} \mathrm{cfu} / \mathrm{g}\right)$ as detailed in the European regulations [6]. From venue $A$, samples from seven different dishes (none of them were chicken or lamb dishes) were analysed, and in three of these Enterobacteriaceae and $B$. cereus were detected at levels exceeding acceptable thresholds [7]. However, these findings remain inconclusive, as these items were collected more than 72 hours after the weddings. No C. perfringens was isolated in any leftover food examined.

Environmental samples

High levels of Enterobacteriaceae and B. cereus were also found in environmental samples taken at the caterer's preparation premises. In addition, one out of three hand swabs, from the chef who had prepared the Jeera chicken and Lamb karahi for the weddings, tested positive for enterococci, Enterobactericeae and E. coli. The hand swabs were not tested for $C$. perfringens.

\section{Stool samples from chefs}

Stool samples were available for four of the kitchen chefs. None of them had gastrointestinal symptoms and all tested negative for oocytes, parasites, Salmonella, Shigella, Campylobacter, C. perfringens, and Staphylococcus aureus using standard isolation methods.

\section{Kitchen inspection}

The environmental health officers visited the caterer's premises on numerous occasions during the outbreak investigation. In addition to the samples mentioned above, some samples of food stored in the kitchen (but not used in the food for the two weddings) were taken: A garlic and ginger paste and a not fully processed paneer cheese were both found to have above threshold levels of Enterobactericeae and E. coli. In addition to the poor kitchen hygiene, the environmental health officers found that none of the staff were adequately trained in food hygiene and that temperature control during food handling was inadequate. 
TABLE 1

Single-variable analysis of all food and drinks items consumed at the two venues of the outbreak, London, July 2009 (merged analysis)

\begin{tabular}{|c|c|c|c|c|c|c|c|}
\hline & \multicolumn{2}{|c|}{ Cases } & \multicolumn{2}{|c|}{ Controls } & \multirow[b]{2}{*}{ OR } & \multirow[b]{2}{*}{$95 \% \mathrm{Cl}$} & \multirow[b]{2}{*}{ P value } \\
\hline & Exposed & Not exposed & Exposed & Not exposed & & & \\
\hline Chicken tikka $^{a}$ & 79 & 14 & 28 & 13 & 2.62 & $1.11-6.18$ & 0.027 \\
\hline Lamb karahi $^{\mathrm{a}}$ & 69 & 22 & 23 & 18 & 2.45 & $1.13-5.33$ & 0.022 \\
\hline Fish pakoraa & 67 & 24 & 28 & 13 & 1.3 & $0.58-2.88$ & 0.528 \\
\hline Vegetable Pakora & 43 & 49 & 6 & 35 & 5.12 & $2.01-12.99$ & $<0.0001$ \\
\hline Shish kebab & 43 & 49 & 8 & 33 & 3.62 & $1.53-8.52$ & 0.003 \\
\hline Jeera chicken & 48 & 43 & 4 & 37 & 10.33 & $3.53-29.96$ & $<0.0001$ \\
\hline Sag paneer & 35 & 56 & 6 & 35 & 3.65 & $1.42-9.29$ & 0.006 \\
\hline Chicken Biryani & 50 & 42 & 11 & 30 & 3.25 & $1.47-7.17$ & 0.003 \\
\hline Onion kucha & 9 & 74 & 2 & 37 & 2.25 & 0.52 -infinite & 0.304 \\
\hline Lamb tikka & 35 & 57 & 18 & 23 & 0.78 & $0.37-1.64$ & 0.524 \\
\hline Samosa & 27 & 65 & 16 & 25 & 0.65 & $0.30-1.39$ & 0.271 \\
\hline Aloo tikki & 28 & 63 & 15 & 24 & 0.71 & $0.33-1.54$ & 0.393 \\
\hline Chicken karahi & 25 & 66 & 15 & 26 & 0.66 & $0.30-1.43$ & 0.292 \\
\hline Mixed vegetable curry & 19 & 72 & 7 & 34 & 1.28 & $0.50-3.26$ & 0.611 \\
\hline Bombay aloo & 19 & 73 & 12 & 29 & 0.63 & $0.27-1.44$ & 0.278 \\
\hline Daal tarka & 24 & 66 & 14 & 27 & 0.7 & $0.32-1.54$ & 0.382 \\
\hline Lamb biryani & 32 & 60 & 17 & 24 & 0.75 & $0.36-1.59$ & 0.461 \\
\hline Green seasoning sauce ${ }^{a}$ & 20 & 70 & 6 & 35 & 1.67 & $0.63-4.39$ & 0.313 \\
\hline Red seasoning sauce ${ }^{a}$ & 24 & 66 & 4 & 37 & 3.36 & $1.13-9.95$ & 0.029 \\
\hline Plain roti & 46 & 46 & 9 & 32 & 3.56 & $1.55-8.15$ & 0.002 \\
\hline Cucumber raita $^{a}$ & 22 & 70 & 8 & 33 & 1.3 & $0.53-3.15$ & 0.575 \\
\hline Salads and pickles ${ }^{\mathrm{a}}$ & 31 & 61 & 20 & 21 & 0.53 & $0.25-1.12$ & 0.098 \\
\hline Ras malaia $^{a}$ & 45 & 48 & 13 & 28 & 2.02 & $0.94-4.33$ & 0.073 \\
\hline Gajar halwa $^{a}$ & 36 & 56 & 14 & 27 & 1.24 & $0.58-2.65$ & 0.584 \\
\hline Milk-based drinks & 3 & 78 & 1 & 35 & 1.35 & 0.18 -infinite & 0.799 \\
\hline Tap water & 17 & 63 & 9 & 27 & 0.81 & $0.33-2.00$ & 0.654 \\
\hline Fruit juice & 22 & 60 & 14 & 22 & 0.58 & $0.25-1.31$ & 0.19 \\
\hline Iced drinks & 13 & 67 & 6 & 30 & 0.97 & $0.35-2.70$ & 0.955 \\
\hline
\end{tabular}

$\mathrm{Cl}$ : confidence interval; OR: odds ratio.

${ }^{a}$ Items which were served in both venues.

Note that the number of exposed persons may not add up to $100 \%$ because of missing data.

\section{TABLE 2}

Logistic regression model of the implicated food items consumes at the two venues of the outbreak, London, July 2009 (merged analysis)

\begin{tabular}{|c|c|c|c|c|}
\hline & OR & $95 \% \mathrm{Cl}$ & p (Wald test) & p (LR test) \\
\hline Jeera chicken & 11.52 & $3.70-35.86$ & $<0.0001$ & $<0.00001$ \\
\hline Lamb karahia & 2.71 & $1.11-6.58$ & 0.03 & 0.03 \\
\hline \multicolumn{5}{|c|}{ Variables not included in the model } \\
\hline Chicken tikka ${ }^{a}$ & & & & 0.08 \\
\hline Vegetable pakora & & & & 0.08 \\
\hline Shish kabab & & & & 0.35 \\
\hline Sag paneer & & & & 0.95 \\
\hline Chicken biryani & & & & 0.13 \\
\hline Plain roti & & & & 0.62 \\
\hline Red seasoning sauce ${ }^{a}$ & & & & 0.63 \\
\hline
\end{tabular}

$\mathrm{Cl}$ : confidence interval; LR: likelihood ratio; OR: odds ratio.

${ }^{a}$ Items which were served in both venues.

Variables were included in the model if p<0.05 in the LR test. 


\section{Discussion}

This paper presents the findings of a point-source outbreak linking two weddings and one caterer in three London boroughs. There is strong evidence that a meal at either of the two venues was associated with becoming ill with diarrhoea and vomiting. There is no evidence that this outbreak was the result of humanto-human transmission. The epidemiological analyses as well as the biological plausibility (e.g. incubation time, clinical picture and duration of illness) suggest that $C$. perfringens was the likeliest causative agent [3], and the detection of $C$. perfringens enterotoxin in stool samples of two of the cases supports this conclusion. It should be noted that few stool specimens from the cases were available for testing and that C. perfringens enterotoxin is only detectable in stools up to two days after illness onset [8]. Although outbreaks related to $C$. perfringens are occasionally reported in the UK, these have become increasingly rare in developed countries, often attributed to improved temperature control in kitchens, but also due to mild symptoms and subsequent underreporting of illness [9].

The multivariate analysis of food items demonstrated a significant association between the consumption of Jeera chicken or Lamb karahi and illness. Although we cannot exclude that other dishes may have been contaminated with C. perfringens, it is likely that these two dishes will have contained high numbers of enterotoxigenic $C$. perfringens. Both are curry-based dishes that were prepared together in one common process by the same chef. The high levels of contamination with faecal organisms isolated from the hand swabs of this chef raise the possibility of insufficient personal hygiene as a risk factor for this outbreak. Although no $C$. perfringens was detected in the stool samples from the chefs, it should be noted that $C$. perfringens carrying the enterotoxin gene has been found in healthy food handlers, but only with specialist isolation methods and not routine methods as were used here [5].

Of those exposed to Jeera chicken or Lamb karahi, 92\% and $75 \%$, respectively, became ill, but $95 \%$ of those who consumed both of these dishes became ill; it is possible that this reflects a dose response.

A blast chiller is normally used for cooling large quantities of food quickly by this particular caterer; however it was not being used appropriately at the time of the incident. Temperature control of foods during preparation, cooling, transportation and reheating was poor. Furthermore, the vans used for food transport had no refrigeration and these events took place in July. The evidence of insufficient hygiene, cooling and reheating at the catering company during transport and at both venues (according to environmental health department inspections) are in keeping with a toxin-related gastroenteritis outbreak, including $C$. perfringens enterotoxin $[4,9,10]$. As the distance from the caterer to venue $B$ was substantially longer than to venue $A$, the lack of adequate temperature control during transport may have led to a higher infective dose in venue $B$, which could explain the shorter incubation time and higher proportion of cases with vomiting.

We present the results of a pragmatic outbreak investigation. Its strengths and weaknesses are defined by the rapid investigation required for the public health response, the need for coordination across multiple organisations and the difficulties in contacting attendees at large functions.

The study would have benefited from more controls, and this would have increased the statistical power to detect rare risk exposures. The absence of guest lists, the need for rapid investigations, and the high attack rate made it difficult to recruit additional controls. It is possible that a larger sample size with more controls would have led to increased effect sizes of associations between food items and illness, but it is unlikely that more controls would have altered the main results, because of the high effect sizes observed.

One of the main challenges in this investigation was the lack of appropriate food samples from the weddings and the difficulty in obtaining stool samples from cases, who came from all over the UK. The scarcity of, and delay in obtaining, microbiological samples, including food, stool and environmental samples, illustrates some of the challenges to coordinate actions across multiple organisations in several districts or regions. The decentralised testing of microbiological samples with environmental health officers from different boroughs ordering different tests led to delays. Despite these limitations the epidemiological and microbiological evidence is sufficient to establish a strong association between consumption of the aforementioned food items and subsequent gastrointestinal illness.

The outbreak investigation was conducted rapidly and in a timely fashion, enabling quick implementation of control measures and also minimising recall bias in the interviews. Real-time updates on the results were presented to the outbreak control team and informed further sampling and public health measures. This was partly due to an already prepared outbreak investigation tool kit, including previously tested questionnaires that were available at the North West London Health Protection Unit. The caterer was served with a Hygiene Emergency Prohibition Notice. This required the production of a food safety risk assessment according to specific Hazard Analysis and Critical Control Points (HACCP) before any catering could take place. The caterer complied with the control measures, employed a food hygiene consultant to oversee the food preparation, and produced specific HACCPs for each food product. The company was therefore allowed to continue catering for events despite the prohibition order.

In summary, we report the results of the microbiological and epidemiological investigation of a large pointsource $C$. perfringens enterotoxin outbreak occurring 
simultaneously at two weddings. The outbreaks were associated with consumption of specific curry-based dishes provided by the same caterer. The preparedness and collaboration between different stakeholders enabled real-time availability of investigation results and helped to control this outbreak quickly with a proportionate response.

\section{Acknowledgements}

We wish to acknowledge the Health Protection Agency, London Food and Water Laboratory for the microbiological analysis of food samples associated with the incidents described in this paper.

\section{References}

1. Brynestad S, Granum PE. Clostridium perfringens and foodborne infections. Int J Food Microbiol. 2002; 74(3):195-202.

2. Health Protection Agency (HPA) [Internet]. General outbreaks of Clostridium perfringens gastroenteritis reported to the Health Protection Agency Centre for Infections England and Wales, 1992 - 2006. London: HPA. [Accessed 17 June 2010]. Available from: http://www.hpa.org.uk/Topics/InfectiousDiseases/ InfectionsAZ/ClostridiumPerfringens/EpidemiologicalData/ cperf_DataEw/

3. Heymann DL, editor. Clostridium perfringens food intoxication. Control of Communicable Diseases Manual. Washington D.C.: American Public Health Association, 2004: 214-16.

4. Taormina PJ, Dorsa WJ. Growth potential of Clostridium perfringens during cooling of cooked meats. J Food Prot. 2004; 67(7):1537-47.

5. Heikinheimo A, Lindström M, Granum PE, Korkeala H. Humans as reservoir for enterotoxin gene--carrying Clostridium perfringens type A. Emerg Infect Dis. 2006; 12(11):1724-9.

6. European Commission. Commission Regulation (EC) No $2073 / 2005$ of 15 November 2005 on microbiological criteria for foodstuffs. Official Journal of the European Union. Luxembourg: Publications Office of the European Union. 22.12.2005:L 338/1. Available from: http://eur-lex.europa.eu/ LexUriServ/LexUriServ.do?uri=0J:L:2005:338:0001:0026:EN: PDF

7. Gilbert RJ, de Louvois J, Donovan T, Little C, Nye K, Ribeiro $C D$, et al. Guidelines for the microbiological quality of some ready-to-eat foods sampled at the point of sale. PHLS Advisory Committee for Food and Dairy Products. Commun Dis Public Health. 2000; 3(3):163-7.

8. Berry PR, Rodhouse JC, Hughes S, Bartholomew BA, Gilbert RJ. Evaluation of ELISA, RPLA, and Vero cell assays for detecting Clostridium perfringens enterotoxin in faecal specimens. J Clin Pathol. 1988; 41(4):458-61.

9. Todd EC. Epidemiology of foodborne diseases: a worldwide review. World Health Stat Q. 1997; 50(1-2):30-50.

10. Kalinowski RM, Tompkin RB, Bodnaruk PW, Pruett WP Jr. Impact of cooking, cooling, and subsequent refrigeration on the growth or survival of Clostridium perfringens in cooked meat and poultry products. J Food Prot. 2003; 66(7):1227-32. 